\title{
Field-aligned currents and ionospheric parameters deduced from EISCAT radar measurements in the post-midnight sector
}

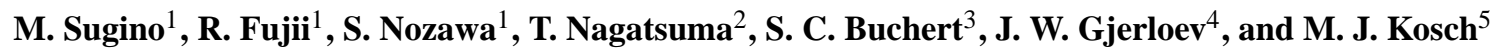 \\ ${ }^{1}$ Solar-Terrestrial Environment Laboratory, Nagoya University, Chikusa-ku, Nagoya 464-8601, Japan \\ ${ }^{2}$ Communications Research Laboratory, 4-2-1 Nukui-kita, Koganei, Tokyo 184-8795, Japan \\ ${ }^{3}$ Swedish Institute of Space Physics, Uppsala Division, Box 537, 75121 Uppsala, Sweden \\ ${ }^{4}$ National Research Council, NASA/Goddard Space Flight Center, Building 2, CODE 696, Greenbelt, MD 20771, USA \\ ${ }^{5}$ Department of Communications Systems, Lancaster University, Lancaster. LA1 4YR, UK
}

Received: 21 February 2002 - Revised: 20 June 2002 - Accepted: 2 July 2002

\begin{abstract}
Attempting to derive the field-aligned current (FAC) density using the EISCAT radar and to understand the role of the ionosphere on closing FACs, we conducted special radar experiments with the EISCAT radar on 9 October 1999. In order to derive the gradient of the ionospheric conductivity $(\operatorname{grad} \Sigma)$ and the divergence of the electric field (div $\boldsymbol{E}$ ) nearly simultaneously, a special experiment employed an EISCAT radar mode which let the transmitting antenna sequentially point to four directions within $10 \mathrm{~min}$; two pairs of the four directions formed two orthogonal diagonals of a square.
\end{abstract}

Our analysis of the EISCAT radar data disclosed that $\Sigma_{P}$ $\operatorname{div} \boldsymbol{E}$ and $\boldsymbol{E} \cdot \operatorname{grad} \Sigma_{P}$ produced FACs with the same direction inside a stable broad arc around 05:00 MLT, when the EISCAT radar presumably crossed the boundary between the large-scale upward and downward current regions. In the most successfully observed case, in which the conductances and the electric field were spatially varying with little temporal variations, the contribution of $\Sigma_{P}$ div $\boldsymbol{E}$ was nearly twice as large as that of $\boldsymbol{E} \cdot \operatorname{grad} \Sigma_{P}$. On the other hand, the contribution of $(\boldsymbol{b} \times \boldsymbol{E}) \cdot \operatorname{grad} \Sigma_{H}$ was small and not effective in closing FACs.

The present EISCAT radar mode along with auroral images also enables us to focus on the temporal or spatial variation of high electric fields associated with auroral arcs. In the present experiment, the electric field associated with a stable arc was confined in a spatially restricted region, within $\sim 100 \mathrm{~km}$ from the arc, with no distinct depletion of electron density. We also detected a region of the high arcassociated electric field, accompanied by the depletion of electron density above $110 \mathrm{~km}$. Using auroral images, this region was identified as a dark spot with a spatial scale of over $150 \times 150 \mathrm{~km}$. The dark spot and the electron depletion were likely in existence for a limited time of a few minutes.

Correspondence to: M. Sugino

(msugino@stelab.nagoya-u.ac.jp)
Key words. Ionosphere (auroral ionosphere; electric fields and currents; particle precipitation)

\section{Introduction}

The field-aligned current (FAC) plays a crucial role in the transfer of momentum and energy between the solar wind, the magnetosphere, and the ionosphere. Satellite observations have revealed its global distribution (e.g. Iijima and Potemra, 1976) and have also enabled us to determine the generation mechanisms of FACs in the magnetosphere (see a review by Iijima, 2000). On the other hand, observations from the ground, especially using incoherent scatter (IS) radars, can provide the temporal and spatial variations of ionospheric currents connecting FACs. If spatial distributions of the ionospheric conductances and electric fields, i.e. the gradient of the ionospheric conductances and the divergence of the electric fields, are known, then the FAC density is fully determined. In order to understand how the ionospheric current is connected as a closure current to the FAC, ground-based observations covering a wide area of the ionosphere are indispensable. Based on EISCAT radar experiments, the aim of this study is to better understand the ionospheric current closure of FACs and the relative contribution of the electric field and the ionospheric conductance to the FAC.

Several studies have attempted to determine the characteristics of FACs deduced from ground measurements. Using a quiet-time model of the ionospheric conductance and measurements of the electric field by the IS radar at Millstone Hill, Yasuhara et al. (1982) estimated the global distribution of FACs on two quiet days. They suggested that the large-scale FACs were controlled mainly by the electric field and not as strongly by the ionospheric conductances. Using a real conductance measurement with the EISCAT radar in the meridian scanning mode, Caudal (1987) deduced the 


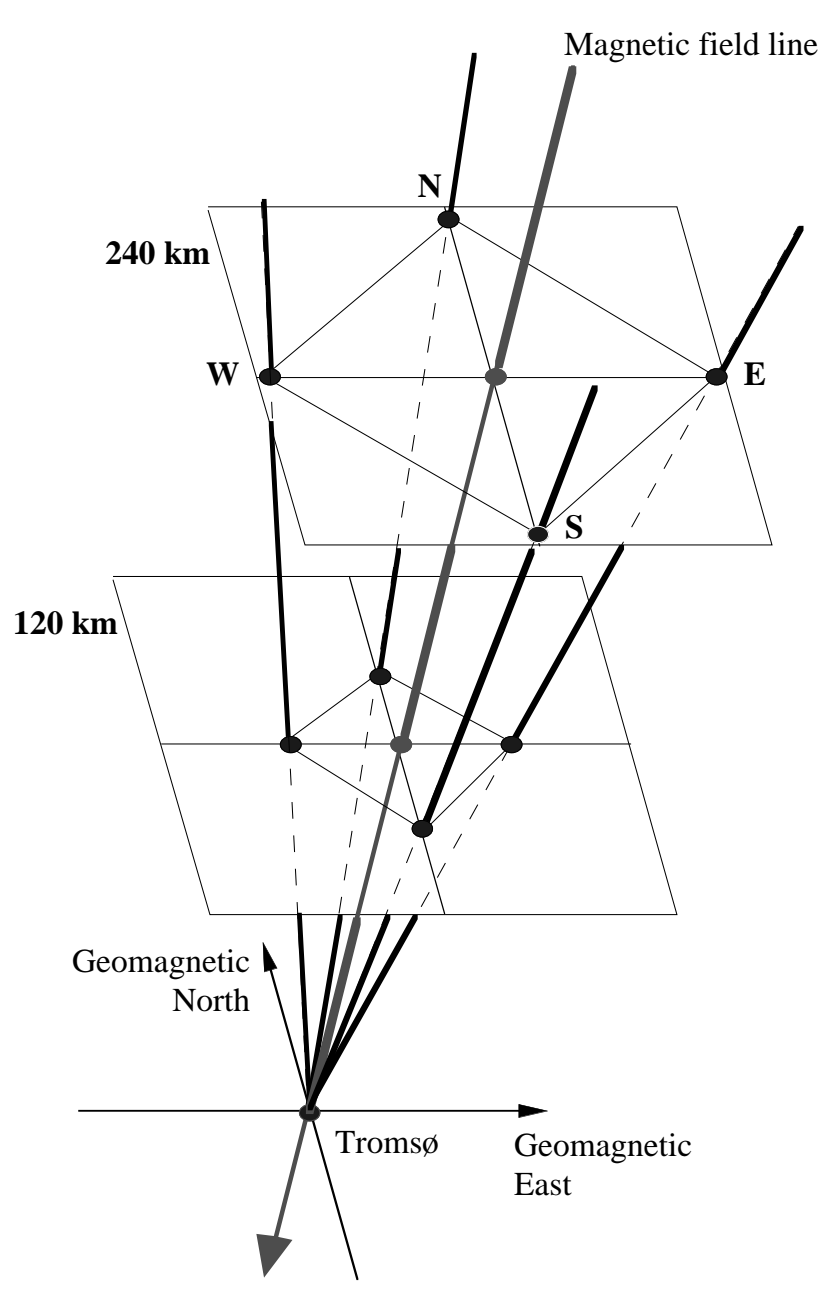

Fig. 1. An illustration of the idealized EISCAT radar mode. The transmitting antenna is sequentially pointed to four positions. Ideally, two pairs of the four directions would form the two orthogonal diagonals of a square. In each of the four positions, the electric field in the F-region $(240 \mathrm{~km})$ is measured with three antennas, and the Pedersen and Hall conductivities are determined along the Troms $\varnothing$ beam.

distribution of FACs on a summer day, which was in general agreement with that derived statistically by Iijima and Potemra (1976). Sorting by the geomagnetic activity index $K_{p}$, Fontaine and Parmirat (1996) showed statistical distributions of the horizontal currents and FACs that were based on models of the large-scale convection (Senior et al., 1990) and conductance (Senior, 1991) derived statistically from observations with the EISCAT radar.

These studies based on IS radar measurements are mainly concerned with the large-scale global distribution of FACs. However, they employed meridian scanning modes, and the Earth's rotation was utilized for obtaining longitudinal distributions. Thus, the global distribution obtained can be justified only when it has negligibly small temporal variations. In order to understand more accurately how the FAC is connected to its closure current and the relative contribution of ionospheric parameters to the FAC, a two-dimensional mea- surement within the time scale of the variations is essential. Also, differences in the Pedersen and Hall current contributions to FACs are of great interest.

Based on an algorithm by Amm (1998), Kosch et al. (2000, 2001) showed the FAC distribution of a plasma flow vortex associated with the brightening of an auroral arc. They illustrated that the downward current region resulted mostly from diverging horizontal Pedersen currents. Assuming a Hall-to-Pedersen conductance ratio, they estimated conductances from the Scandinavian Magnetometer Array (SMA) (Küppers et al., 1979) and Scandinavian Twin Auroral Radar Experiment (STARE) (Greenwald et al., 1978) data. Although STARE is a powerful tool to obtain two-dimensional maps of the electric field, it can provide reliable ionospheric electric fields only when they exceed $15 \mathrm{mV} / \mathrm{m}$ (Cahill et al., 1978). As shown by several studies, the conductance and the electric field closely interact with each other (e.g. de la Beaujardière et al., 1981; Baumjohann, 1983; Marklund, 1984). Hence, it is indispensable to measure these parameters simultaneously and precisely for a better understanding of the three-dimensional current systems.

A merit of using the EISCAT radar is that we can accurately measure temporal and spatial variations of both the conductance and the electric field. In order to determine how the ionospheric current is connected to the FAC quantitatively, we made a special experiment with the EISCAT radar on 9 October 1999. Since the proposed method to determine the FAC must be validated as to whether it provides a meaningful current density or direction, a comparison study based on simultaneous observations with other instruments is important. We present a comparative study using simultaneous observations, such as auroral images and satellite data.

An enhancement of the ionospheric electron density or the Pedersen conductivity is generally seen in an upward FAC region due to auroral precipitation. The paired downward FAC is expected to require a larger ionospheric electric field than the upward FAC, to close the ionospheric current connecting different Pedersen conductivity regions. Low-altitude satellite observations show that some kind of upward FACs with intense precipitation are associated with smaller electric fields; sometimes the electric field appears to be even short-circuited (e.g. Hoffman, 1989). This relatively small electric field is usually considered to be the combination of the magnetospheric electric field and the polarization electric field produced by the charge accumulation due to the inhomogeneity of the ionospheric conductivity (e.g. Baumjohann et al., 1983). It should be emphasized that the polarization electric field, arising from the density inhomogeneity in the polar ionosphere, can drive FACs of ionospheric origin. The polarization electric field can play a role in the re-distribution of the electric field and hence, the modification of the current systems (e.g. Lysak, 1990). Although the development of the polarization electric field is discussed theoretically, it has not been well understood observationally. Thus, the investigation of the electric fields associated with auroral arcs also enables us to understand how ionospheric closure currents and FACs are distributed around auroral arcs. 


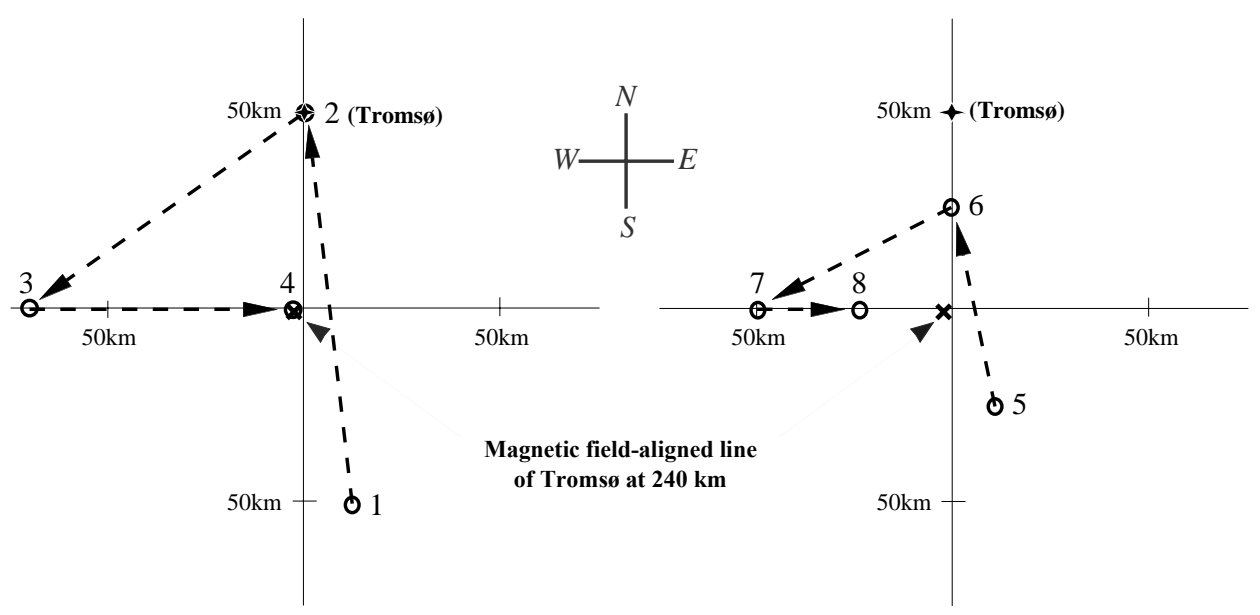

Fig. 2. Beam positions at $240 \mathrm{~km}$ height in the mode employed, taking into account the Earth's rotational effect. The spatial scale for electric field measurements at $240 \mathrm{~km}$ height is $100 \mathrm{~km}$ in the first set of beams (1-2-3-4), and $50 \mathrm{~km}$ in the second set (5-6-7-8). The spatial scale for the conductance measurement becomes $50(25) \mathrm{km}$ in the first (second) set.

Kosch et al. (2000) showed the region of diverging ionospheric electric fields associated with a sudden brightening of the arc. They suggested that the region corresponded to a downward FAC and black auroras; however, no black auroras could be identified in their all-sky TV data, owing to its limited resolution. Using an all-sky camera and the EISCAT radar, Lewis et al. (1994) revealed the presence of a band of enhanced electric field on only one side of a drifting auroral arc, but its development was not detected by all-sky camera images. Although several studies have reported the characteristics of a region with an enhanced electric field, its temporal development is still not clear. Based on both EISCAT radar measurements and auroral images, we also focus on the temporal or spatial variation of the region with high electric fields associated with auroral arcs.

\section{EISCAT radar mode employed}

In this study we have used the tristatic European Incoherent Scatter (EISCAT) radar, the so-called Kiruna-SodankyläTroms $\varnothing$ (KST) UHF radar system (Folkestad et al., 1983; Rishbeth and Williams, 1985). Scattered signals from the radar beam transmitted at Troms $\varnothing\left(69.6^{\circ} \mathrm{N}, 19.2^{\circ} \mathrm{E}, 66.2^{\circ}\right.$ invariant latitude) are received at three stations; Troms $\emptyset$ itself, Kiruna, and Sodankylä, and thus, the three-dimensional ion velocity can be calculated. The electric field $(\boldsymbol{E})$ can be determined from the three-dimensional ion velocity at an altitude ( $240 \mathrm{~km}$ in our mode) in the F-region, where ions move by $\boldsymbol{E} \times \boldsymbol{B}$ drift, since the ion gyrofrequency is much greater than the ion-neutral collision frequency here. The magnetic field $(\boldsymbol{B})$ is derived from the International Geomagnetic Reference Field (IGRF) model (Barton, 1997). Along the Troms $\varnothing$ beams, EISCAT radar measurements also provide the electron density and ion/electron temperatures with a height resolution of about $3 \mathrm{~km}$ in the E-region, and of about $20 \mathrm{~km}$ in the F-region. Thus, the Pedersen and Hall conductivities ( $\sigma_{P}$ and $\sigma_{H}$ ) can be calculated using formulas by Brekke and Hall (1988) and the MSISE-90 neutral atmospheric model (Hedin, 1991). The Pedersen and Hall conductances $\left(\Sigma_{P}\right.$ and $\left.\Sigma_{H}\right)$ are calculated by integrating them over the height range from 90 to $300 \mathrm{~km}$. The adoption of the lower height limit of $90 \mathrm{~km}$ may be justified, except under extremely energetic precipitation, which could produce as much as $15 \%$ of the Hall conductance below $90 \mathrm{~km}$ (Schlegel, 1988). From these ionospheric parameters we can derive the ionospheric current $(\boldsymbol{J})$ as,

$\boldsymbol{J}=\boldsymbol{J}_{P}+\boldsymbol{J}_{H}=\Sigma_{P} \boldsymbol{E}+\Sigma_{H}(\boldsymbol{b} \times \boldsymbol{E})$,

where $\boldsymbol{J}_{P}$ and $\boldsymbol{J}_{H}$ are the height-integrated Pedersen and Hall currents, respectively, and $\boldsymbol{b}$ is the unit vector in the direction of the Earth's magnetic field. The experiment also enables us to determine the relative contribution of the gradient of the ionospheric conductance and the divergence of the electric field to the FAC density $j_{\|}$(positive away from the ionosphere) in the following way,

$$
\begin{aligned}
-j_{\|} & =\operatorname{div} \boldsymbol{J}=\Sigma_{P} \operatorname{div} \boldsymbol{E}+\boldsymbol{E} \cdot \operatorname{grad} \Sigma_{P} \\
& +(\boldsymbol{b} \times \boldsymbol{E}) \cdot \operatorname{grad} \Sigma_{H}+\Sigma_{H} \operatorname{div}(\boldsymbol{b} \times \boldsymbol{E}) .
\end{aligned}
$$

Each of these terms depends on both the electric field and the conductance. The first term depends on the divergence of the electric field, and is called the term of "magnetospheric origin FAC" (e.g. Sofko et al., 1995). The second and third terms, called the terms of "ionospheric origin FAC", depend on the gradient of Pedersen and Hall conductances, respectively. Since our method assumes that the ionospheric electric field is a curl-free potential electric field ( $\operatorname{rot} \boldsymbol{E}=0$ ), the last term should be zero.

In order to derive the gradient of the ionospheric conductance and divergence of the electric field, we conducted a special experiment using the EISCAT radar. Figure 1 is an illustration of the idealized EISCAT radar mode. The transmitting antenna is sequentially pointed to four positions, similar 

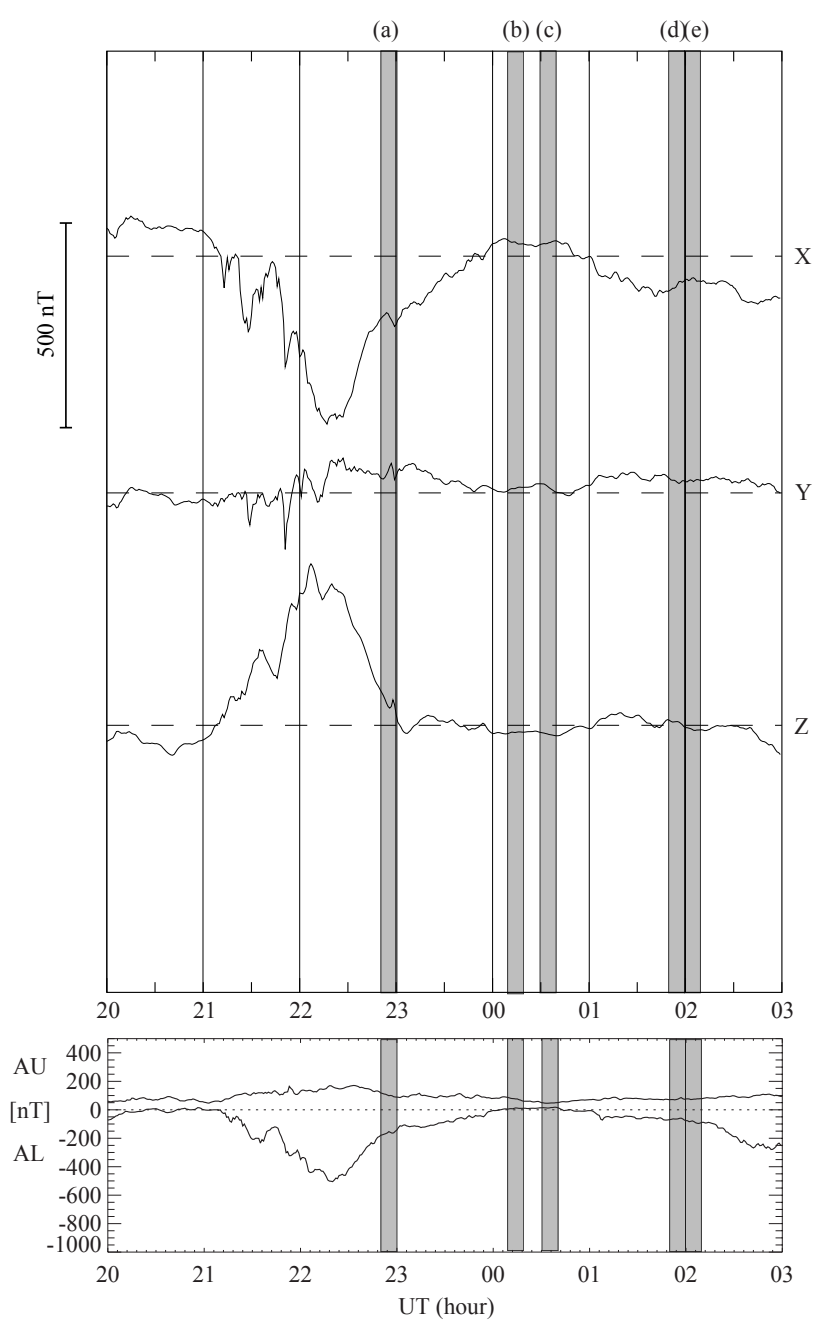

Fig. 3. Ground-based magnetometer data at Troms $\varnothing$ (upper) and the $A U-A L$ index (lower) during our experiment.

to CP-2 mode (see e.g. Collis, 1995). Ideally, two pairs of the four directions would form the two orthogonal diagonals of a square, as illustrated in Fig. 1. In each of the four positions, the electric field in the F-region $(240 \mathrm{~km})$ is measured with the three antennas, and the Pedersen and Hall conductivities are determined along the Troms $\varnothing$ beam.

The Earth rotates once a day under the ionosphere with the rotation speed of about $10 \mathrm{~km} / \mathrm{min}$ at Troms $\varnothing\left(69.6^{\circ} \mathrm{N}\right)$. Taking into account this rotational effect, we designed our radar mode to make the four beam positions form a square in the ionosphere, where the patterns of the electric field (equivalently the plasma convection) and precipitation (the auroral oval) do not move with the Earth's rotation, but rather are fixed in the Sun-Earth coordinates. Figure 2 illustrates the beam positions at $240 \mathrm{~km}$ height in our special radar mode employed. It is noted that the transmitting antenna is directed nearly along the geomagnetic field in the beam position 4. Also, beam 2 points vertically upward. When the transmitting antenna is not directed along the magnetic field line at Troms $\varnothing$, the measurements in the E- (conductances) and F- (electric fields) regions are not on the same magnetic field line. However, this may not be a serious problem if the electric field and the ionospheric conductances are linearly varying in the square region concerned, which is implicitly assumed in this method.

What we are able to derive is, for example, the divergence of the electric field with the spatial scale approximately the same as, or larger than, the spatial size of the square observing region. Contributions from smaller scale variations cannot be taken into account. In our radar mode we alternately measured two sets of the four beam directions, in order to investigate the distributions of the parameters in different spatial scales. As shown in Fig. 2, the spatial scale for electric field measurements at $240 \mathrm{~km}$ height is $100 \mathrm{~km}$ in the first set of beam positions (1-2-3-4), and $50 \mathrm{~km}$ in the second set (5-6-7-8). The height-dependent Pedersen conductivity is typically largest around $120 \mathrm{~km}$ in height. Hence, the adopted spatial scale for the conductance measurement becomes $50 \mathrm{~km}$ in the first set and $25 \mathrm{~km}$ in the second set. The time resolution of the post-integrated parameters is about $2 \mathrm{~min}$. To complete one antenna cycle consisting of four positions requires $10 \mathrm{~min}$, which may allow us, under some conditions, to assume that the measured distribution is a spatial distribution without temporal variations.

\section{Observations}

We conducted our experiment with the EISCAT radar from 20:30 UT on 9 October to 02:30 UT on 10 October 1999. Figure 3 shows the ground-based magnetometer data at Troms $\varnothing$ (upper panel) and $A U-A L$ index (lower panel) during this experiment. A relatively quiet period can be seen, except from 21:00-24:00 UT, when disturbances due to substorms are identified. The geomagnetic disturbance level is $K_{p}=3^{-}$(18:00-21:00 UT on 9 October, 00:00-03:00 UT on 10 October) and $K_{p}=3$ (21:00-24:00 UT on 9 October).

Since our method to calculate a FAC density assumes little temporal variations within $10 \mathrm{~min}$, we selected suitable time periods from the whole experiment interval by checking the digital all-sky imager (DASI) data (Kosch et al., 1998a). Figure 4 shows all-sky images of the selected events we will present later. Little temporal variations are seen, except in case (a). The all-sky camera is located at $69.3^{\circ} \mathrm{N}$, $20.4^{\circ} \mathrm{E}$, about $50 \mathrm{~km}$ from the EISCAT transmitter. The spatial coverage is $67.6-72.6^{\circ} \mathrm{N}, 13.5-26.0^{\circ} \mathrm{E}$ geographic (about $520 \times 520 \mathrm{~km}$ ) with $10 \times 10 \mathrm{~km}$ resolution. The coordinates used here are positions mapped to a $100 \mathrm{~km}$ altitude. The intensity is calibrated in Rayleighs for $557.7 \mathrm{~nm}$. The observation starting time (UT) and beam position corresponding to numbers (1-8) in Fig. 2 are noted in the top of each image of Fig. 4. The white dot near the center of each image is the position of the EISCAT radar beam (not radar site) at $100 \mathrm{~km}$ altitude. Thus, the white dot shows the point where the ionospheric conductance is precisely observed, and it shifts sequentially along with the beam scan- 
Plot time $=150 \mathrm{sec}$

Camera location:

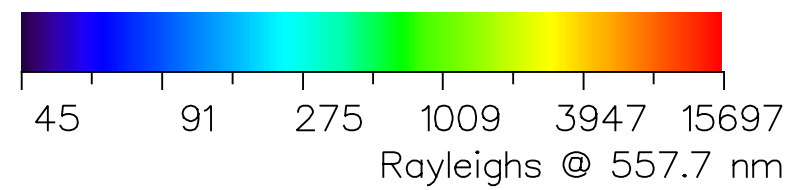

Lat $=69.3$ Long $=20.4$

(a)
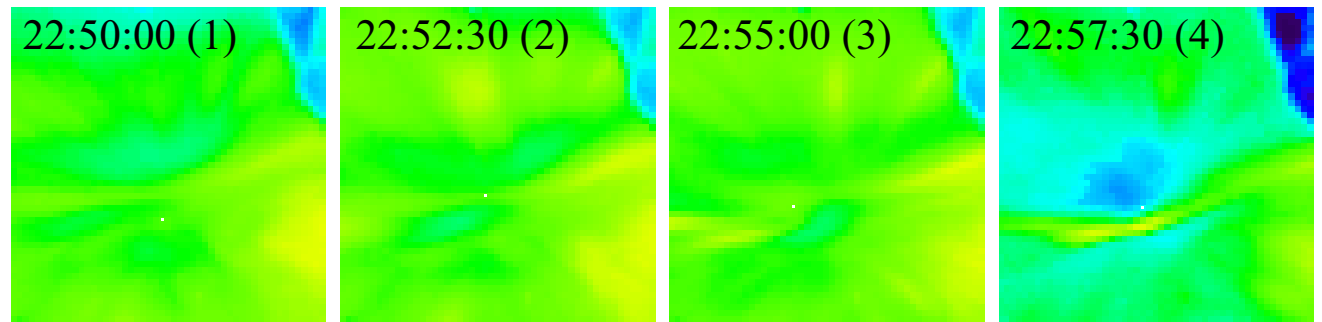

(b)
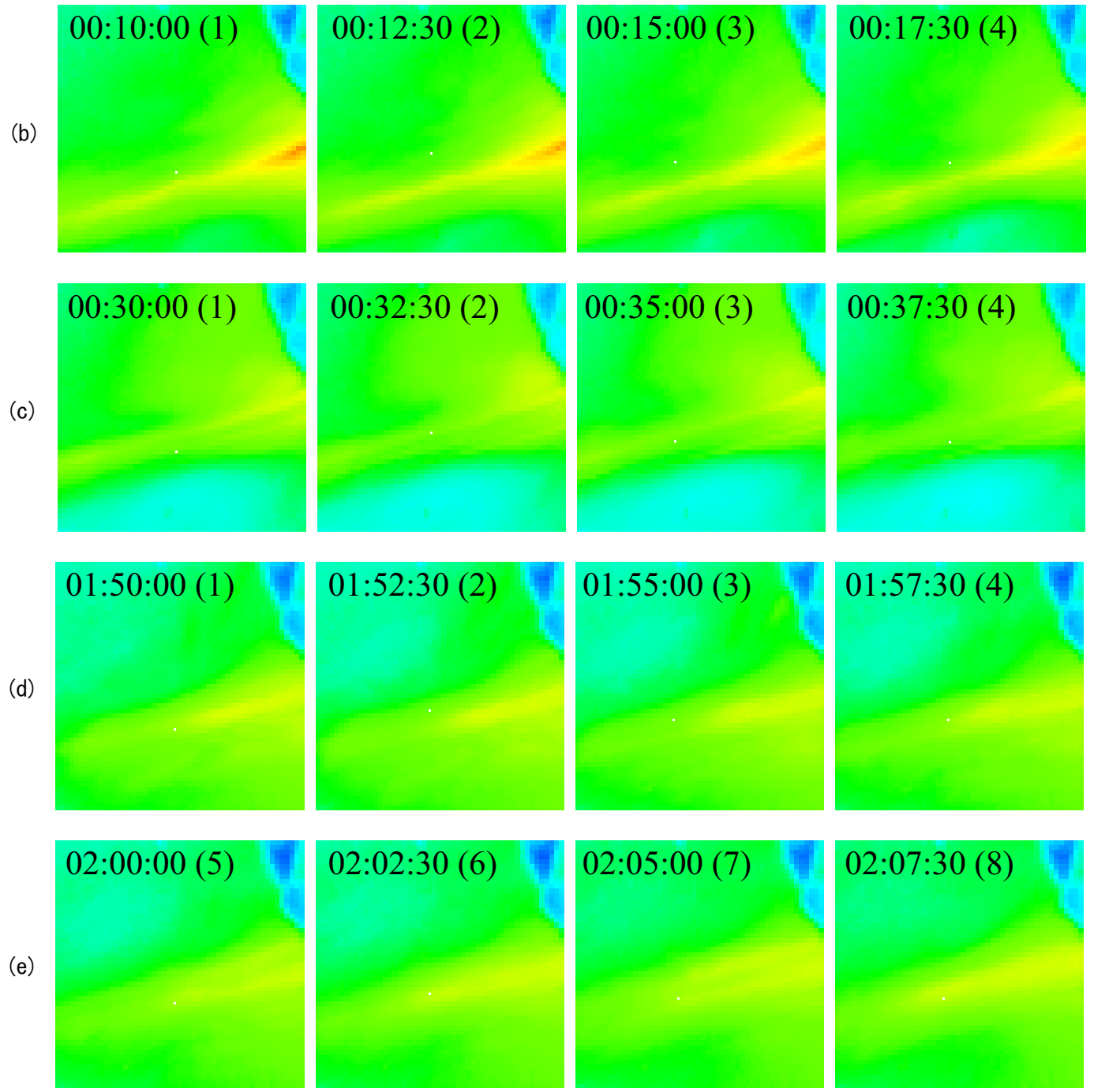

Fig. 4. All-sky images of the selected events. The spatial coverage is $67.6-72.6^{\circ} \mathrm{N}, 13.5-26.0^{\circ} \mathrm{E}$ geographic $($ about $520 \times 520 \mathrm{~km})$ at $10 \times 10 \mathrm{~km}$ resolution. Mapping is for $100 \mathrm{~km}$ altitude. The intensity is calibrated in Rayleighs for $557.7 \mathrm{~nm}$. The white dot near the center of each image is the position of the EISCAT radar beam at $100 \mathrm{~km}$ altitude. The observation starting time (UT) and beam position corresponding to numbers (1-8) are noted in the top of each image. The dark triangle in the top right corner of every image is the mountain near Skibotn, Norway. 


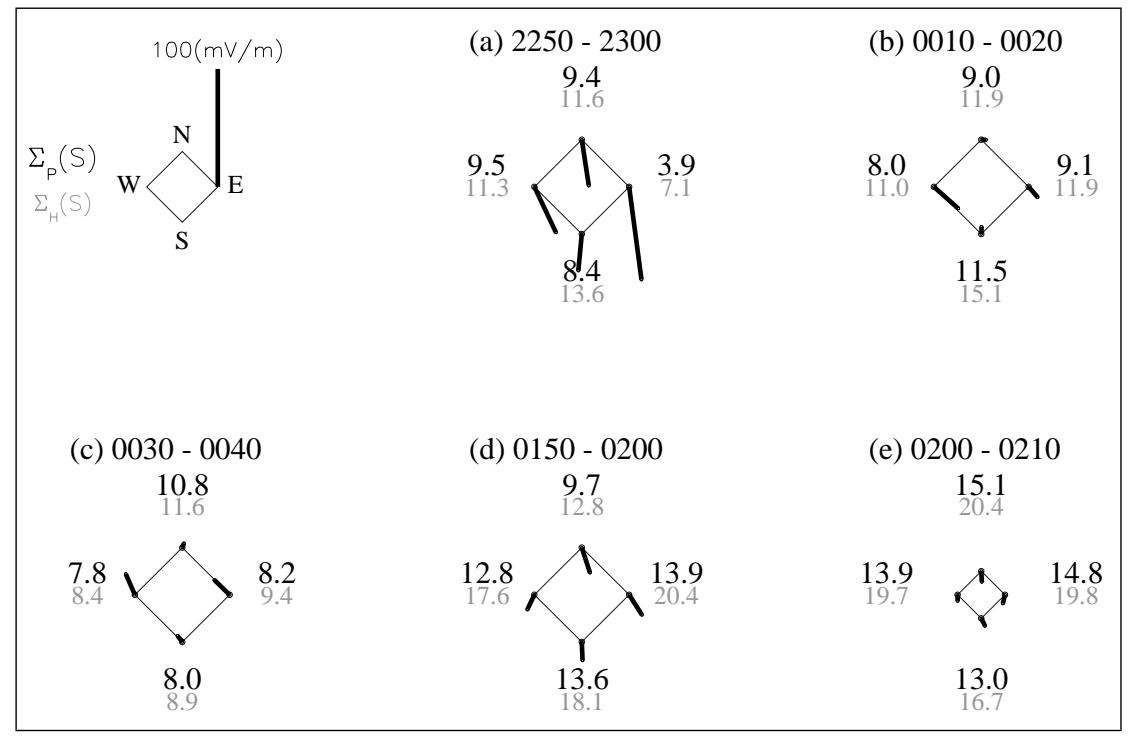

Fig. 5. Spatial variations of the conductance and the electric field observed with the EISCAT radar. Each position of the quadrilateral corresponds to the four beam positions, respectively; bottom (south, beam 1 or 5), top (north, beam 2 or 6), left (west, beam 3 or 7), and right (east, beam 4 or 8 ). Each thick line indicates the strength and direction of the electric field. The Pedersen (upper) and Hall (lower) conductances are denoted in the unit of $\mathrm{S}$.

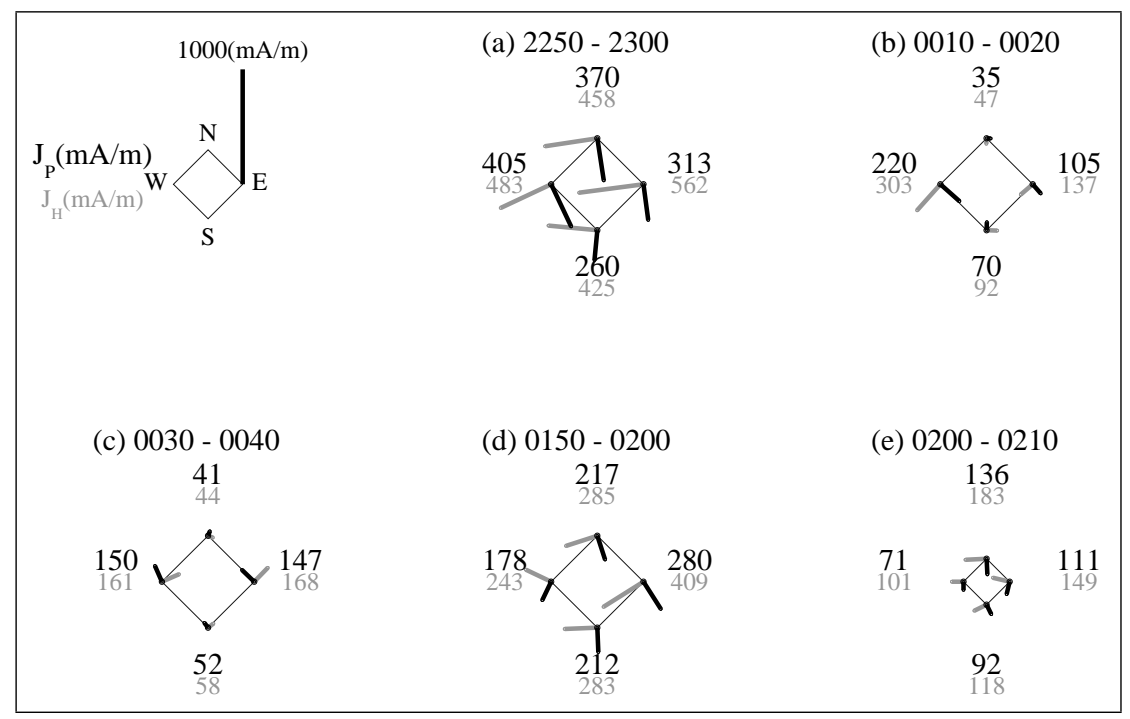

Fig. 6. Spatial variations of the calculated height-integrated Pedersen and Hall currents, assuming that both of the conductance and the electric field are measured on the same magnetic field line. Each thick (thin) line indicates the strength and direction of the Pedersen (Hall) current. ning. The dark triangle in the top right corner on each image is a mountain near Skibotn, Norway.

Figure 5 shows spatial variations of the conductance and the electric field observed with the EISCAT radar in the selected cases corresponding to Fig. 4. Each position of a quadrilateral corresponds to the four beam positions, respectively; bottom (south, beam 1 or 5), top (north, beam 2 or 6 ), left (west, beam 3 or 7), and right (east, beam 4 or 8). Each thick line denotes the strength and direction of the electric field. The Pedersen (upper) and Hall (lower) conductances are also denoted in the unit of S. Note that, except for the beam 4 , the measurements of conductances and electric fields are not on the same magnetic field line. Using an approximation that both the conductance and electric field are measured on the same magnetic field line, we present spatial variations of the calculated height-integrated Pedersen $\left(J_{P}\right)$ and Hall $\left(J_{H}\right)$ currents in Fig. 6. Each thick (thin) line indicates the strength and direction of the Pedersen (Hall) current.

Figure 7 presents the calculated FAC density using the formula (2). Each term (except the last term) of the formula (2) and the total FAC density are plotted. Also, northward and eastward components of $\Sigma_{P}$ div $\boldsymbol{E}$ and $\boldsymbol{E} \cdot \operatorname{grad} \Sigma_{P}$ are shown. A positive value means an upward current density. In order to examine how temporally stable the ionospheric parameters are, rot $\boldsymbol{E}$ is also plotted. Ideally, rot $\boldsymbol{E}$ becomes zero. The greater the amplitude of rot $\boldsymbol{E}$ is, the less valid our assumption becomes.

\section{Results}

In case (a), during 22:50-23:00 UT, a lot of temporal variations are seen in the aurora camera, as depicted in the top four panels of Fig. 4. At first, we will present the cases in which 


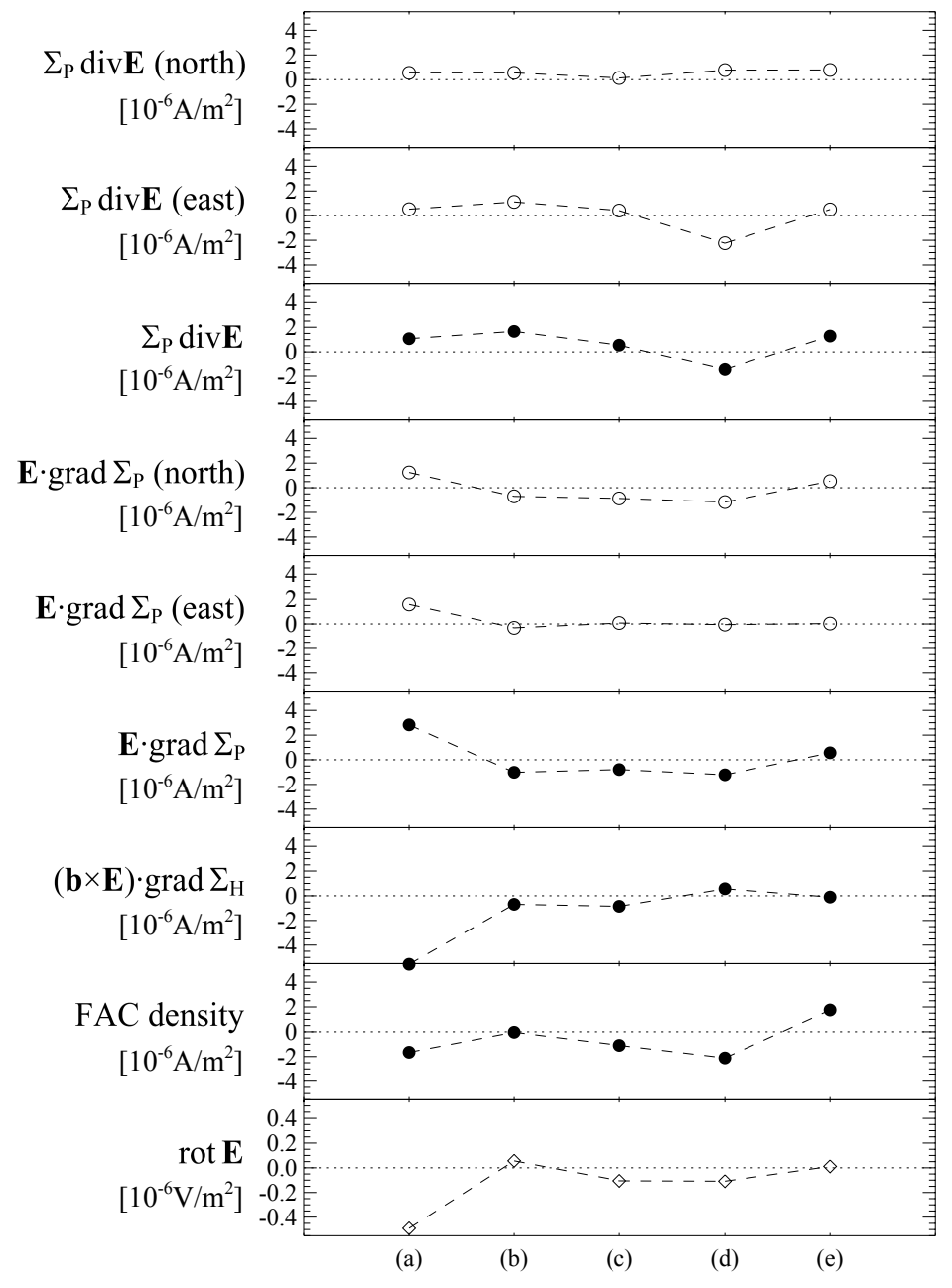

Fig. 7. Calculated FAC densities deduced from EISCAT radar measurements. Each term (except the last term) of the formula (2), the total FAC density, and rot $\boldsymbol{E}$ are plotted. Also, northward and eastward components of $\Sigma_{P}$ $\operatorname{div} \boldsymbol{E}$ and $\boldsymbol{E} \cdot \operatorname{grad} \Sigma_{P}$ are plotted. A positive value means an upward current density. the ionosphere is relatively stable, and then discuss the case (a).

\subsection{Cases (b) 00:10-00:20 UT and (c) 00:30-00:40 UT on 10 October 1999}

The EISCAT radar observed a region around a relatively small-scale auroral arc. Both the ground-based magnetometer data in Fig. 3 and auroral images in Figs. $4 \mathrm{~b}$ and $\mathrm{c}$ indicate that temporal variations are small during these time periods.

During 00:10-00:20 UT in Fig. 5b, the conductance in the south position is larger than that in the other three positions, with a small electric field. An anti-correlation between the conductance and the electric field strength is seen. This has been reported as one of the common features of auroral arcs (e.g. Baumjohann, 1983; Marklund, 1984). Figure 4b confirms that the arc is located at the southward position of the larger square formed by the four beam directions. While the electric field in the north position is also small, with a low conductance, however, this seems due to the large distance between the north position and the arc $(100 \mathrm{~km}$ at $240 \mathrm{~km}$ altitude). This small electric field in the north position implies that the background convection electric field is also small.
On the other hand, in the vicinity neighboring the arc, the electric field is enhanced with a low conductance in the east and west positions.

During 00:30-00:40 UT in Fig. 5c, a conductance enhancement can be seen in the north position with a small electric field. Figure $4 \mathrm{c}$ depicts a narrow arc similar to that shown in Fig. 4b, however, the arc is located in the north of the region measured with the EISCAT radar. Also, in the vicinity of the arc, enhancements of the electric field can be seen.

In summary, during these periods, the relatively high electric field associated with the narrow arc is confined to a spatially restricted region, within $100 \mathrm{~km}$ from the arc. This arc-associated electric field is rather stable. In Fig. 7 for cases (b) and (c), $\Sigma_{P}$ div $\boldsymbol{E}$ produces an upward FAC, while $\boldsymbol{E} \cdot \operatorname{grad} \Sigma_{P}$ produces the opposite direction of the FAC.

\subsection{Cases (d) 01:50-02:00 UT and (e) 02:00-02:10 UT on 10 October 1999}

The EISCAT radar surveyed a region around a broad auroral band. Both ground-based magnetometer data at Troms $\emptyset$ in Fig. 3 and auroral images in Figs. $4 d$ and e suggest that the 


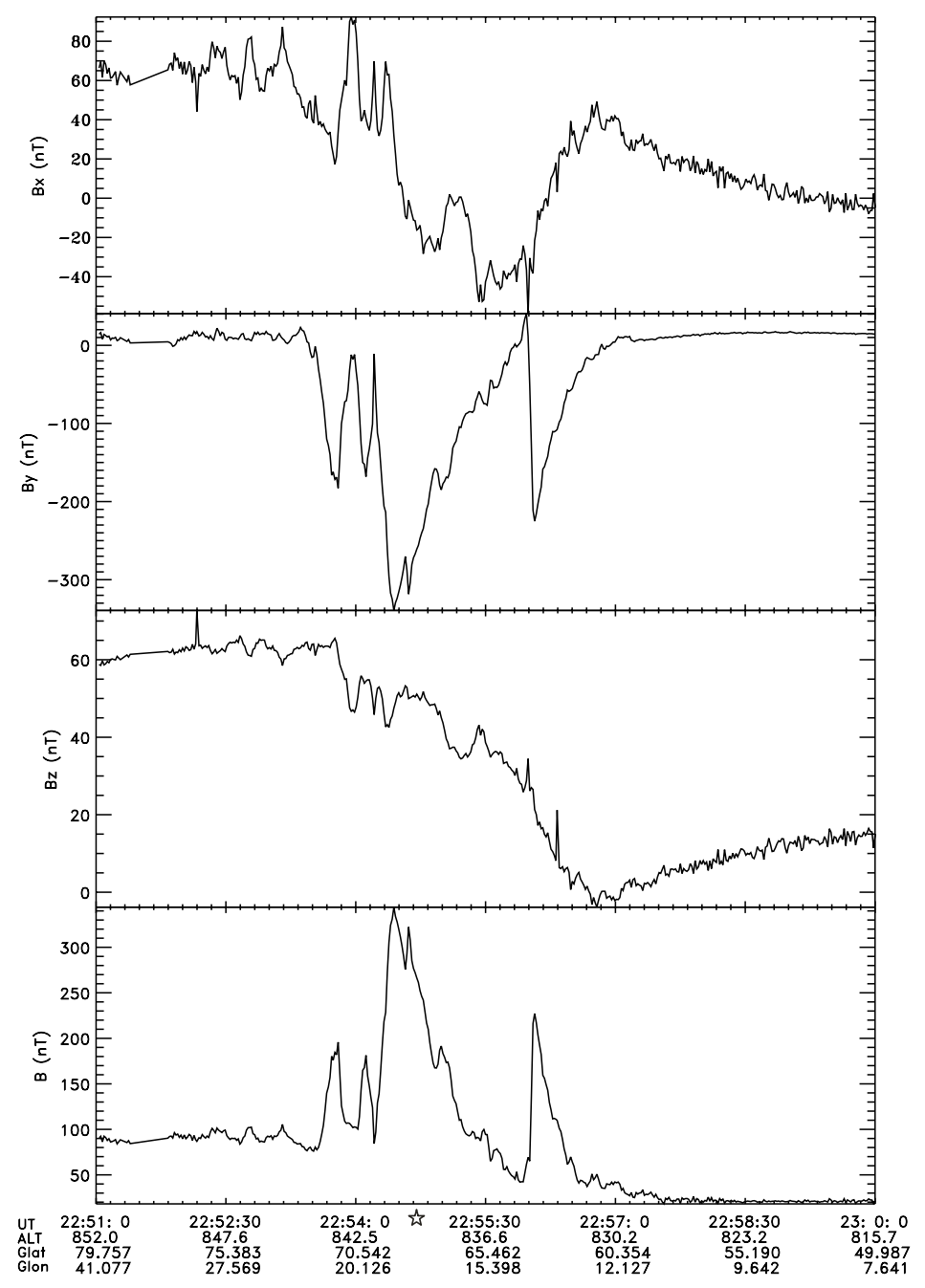

Fig. 8. Magnetic field measurements obtained by the flux-gate magnetometer carried on the Ørsted satellite. $B_{x}$, $B_{y}, B_{z}$ and $B$ are shown after subtraction of the preliminary Ørsted main field model, where $X$ is positive geographic north, $Y$ is positive east, $Z$ is positive down, and $B$ is the total magnetic field strength. The closest position of the EISCAT radar is indicated by the star. temporal variation is also small during these periods. The thickness of the auroral arc is larger compared with the arc seen in the previous cases (b) and (c), and its border is not so well defined. The $A U-A L$ index in Fig. 3 suggests that these cases are during what appears to be a prolonged growth phase of a minor substorm.

During 01:50-02:00 UT, the conductances in the south and east positions are larger than those in the other two positions in Fig. 5d. The electric field is also not small with high conductances. This feature is different from that in the previous cases, which showed an anti-correlation between the conductance and the electric field. Figure 4d suggests that the EISCAT radar sounded a region northwestward of the broad arc.

On the other hand, during 02:00-02:10 UT, the conductivity is high and has the maximum in the north position in Fig. 5e. The electric field is small on each of the four positions, suggesting that the EISCAT radar sounded a region inside of the broad arc. This is confirmed by the auroral images in Fig. 4e. It is noted that, in case (e), the EISCAT radar measured a smaller square region formed by the beam positions 5-8.

As shown in Fig. 7, for cases (d) and (e), a downward FAC appears in the north of the arc during 01:50-02:00 UT, and an upward FAC inside the arc during 02:00-02:10 UT. In cases (d) and (e), both $\Sigma_{P} \operatorname{div} \boldsymbol{E}$ and $\boldsymbol{E} \cdot \operatorname{grad} \Sigma_{P}$ produce the same direction FAC. On the other hand, $(\boldsymbol{b} \times \boldsymbol{E}) \cdot \operatorname{grad} \Sigma_{H}$ is small and not effective in closing the FAC. Hence, the divergence of the electric field and the gradient of the Pedersen conductance play a significant role in closing the FAC through the ionosphere.

In Fig. 5, except in case (a), one notices that all of the stable arcs have a conductivity gradient in the east-west direction and that the conductivity in the east position is higher than that in the west position in each case. Also in Fig. 4, the stable arcs are brighter toward the east. From the viewpoint of the current closure in the ionosphere, however, the conductivity gradient in the north-south direction plays a more crucial role than that in the east-west direction, since the northsouth component of the electric field is generally larger than the east-west component, as seen in Fig. 5. Hence, the term $\boldsymbol{E} \cdot \operatorname{grad} \Sigma_{P}$ is highly dependent on the north-south gradient of the ionospheric Pedersen conductivity. While the term $(\boldsymbol{b} \times \boldsymbol{E}) \cdot \operatorname{grad} \Sigma_{H}$ depends mainly on the east-west gradient of the ionospheric Hall conductivity, this gradient is not so large 

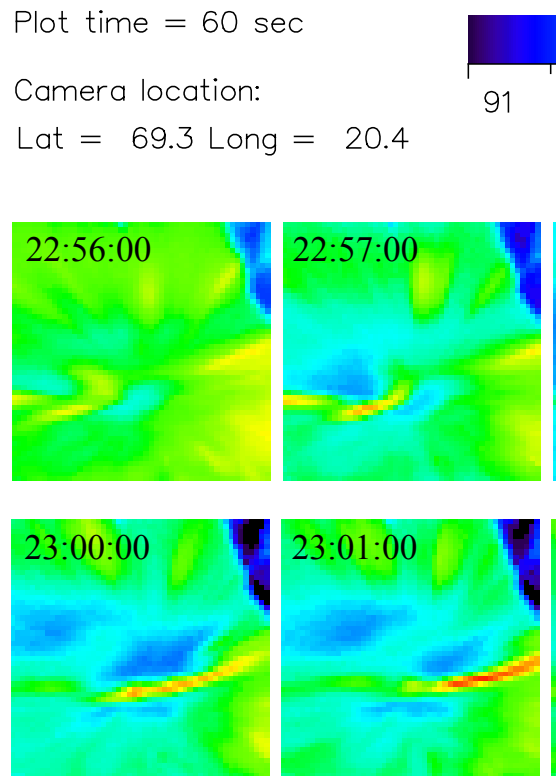
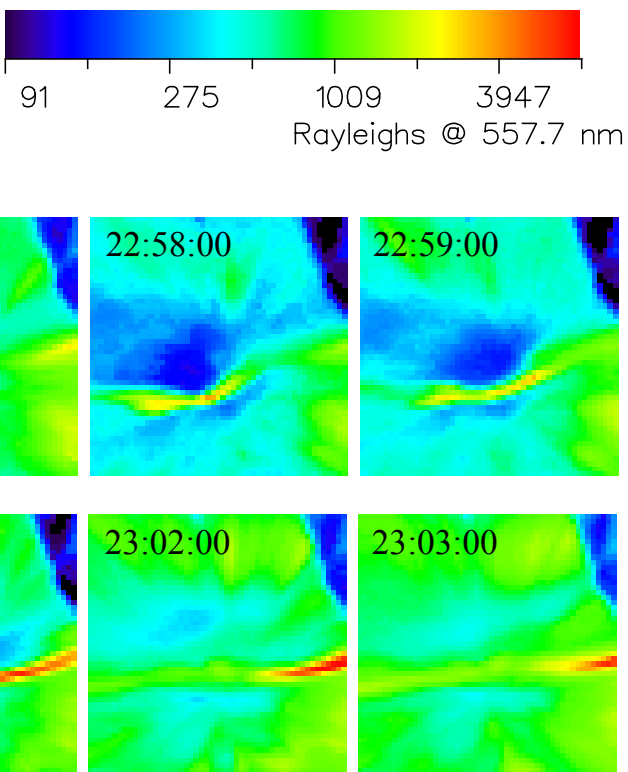

Fig. 9. All-sky images with a time resolution of $1 \mathrm{~min}$, around the time when a dark spot was observed.

compared with the north-south gradient of the Pedersen conductivity, as shown in Fig. 5e. Thus, the FACs close through the Pedersen current rather than through the Hall current in the ionosphere in this specific case.

\subsection{Case (a) 22:50-23:00 UT on 9 October 1999}

During this time period, the Ørsted satellite passed over the EISCAT radar at 22:54 UT. The magnetic field measurements obtained from the fluxgate magnetometer carried on board the Ørsted satellite are shown in Fig. 8. $B_{x}, B_{y}, B_{z}$ and $B$ are magnetic fields after subtraction of the Earth's main fields from the observed magnetic fields, where $X$ is geographic north, $Y$ is east, $Z$ is down, and $B$ is the total magnetic field strength, $B=\left(B_{x}^{2}+B_{y}^{2}+B_{z}^{2}\right)^{1 / 2}$. Notice that the largest perturbations are seen in the $Y$-direction, indicating that the Ørsted trajectory is nearly perpendicular to the FAC sheets. The Ørsted observation indicates that a large-scale downward FAC region is located between about $72.6^{\circ} \mathrm{N}$ and $69.0^{\circ} \mathrm{N}$, while an upward FAC region is located between about $69.0^{\circ} \mathrm{N}$ and $63.5^{\circ} \mathrm{N}$. The nearest position to the EISCAT radar (indicated by the star in Fig. 8) is located at the separation between the two large-scale current sheets. When the Earth rotates and a substorm evolves, the EISCAT radar appears to be shifted back and forth between the two FAC regions. In the vicinity of the EISCAT radar, the Ørsted satellite observes many small-scale FACs within the large-scale FACs. This is a common feature of an auroral substorm during this MLT (e.g. Fujii et al., 1994). The $A U-A L$ index in Fig. 3 also suggests that this case (a) is during the late recovery phase of a substorm.

Several temporal variations are seen in the auroral images, as depicted in Fig. 4a. Especially in the east position (22:58 23:00 UT), the auroral image shows a dark spot adjacent to a narrow bright arc. Correspondingly, the EISCAT radar detects a very high electric field $(\sim 80 \mathrm{mV} / \mathrm{m})$ and low conductances in this position, as exhibited in Fig. 5a. This electric field seems unstable and different from the arc-associated electric fields, as shown in cases (b) and (c), which are rather stable. We have selected this case (a) because a noteworthy dark spot was detected. It should be noted that the FAC calculated from the four position measurements with the EISCAT radar is probably not accurate because this case violates the necessary assumption of no temporal variation over $10 \mathrm{~min}$. This is confirmed by the calculation of $\operatorname{rot} \boldsymbol{E}$, which is much larger than in the other cases shown in Fig. 7.

With a time resolution of $1 \mathrm{~min}$, Fig. 9 depicts the detailed temporal variation of the dark spot around the observed time. The dark spot is clearly identified only during 22:582:300 UT, drifting eastward slowly. Simultaneously, a narrow bright arc appears southward of the dark spot. Also south of the arc, a narrow dark region can be seen during 22:5822:59 UT. We observed another dark spot around 21:46 UT (not shown here) also lasting for a limited time of a few minutes.

Figure 10 shows EISCAT radar measurements of the height distribution of the Pedersen (thick line) or Hall (thin line) conductivity and the electron density. During 22:5823:00 UT, the EISCAT radar sounded inside of the dark spot, as shown in Fig. 4a, with the field-aligned beam position 4 . The low conductances and the enhanced electric field can be seen. The electric field is directed to the narrow arc, and thus, the Pedersen current also flows from the dark spot toward the arc. Above $110 \mathrm{~km}$ altitude, the electron density is depleted. The measurements at several altitudes are missing due to weak backscatters from low electron densities with relatively large errors (as denoted by the crosses in Fig. 10). Such a depletion especially leads to a reduction of the Ped- 
ersen conductivity, since it has a maximum around $120 \mathrm{~km}$ altitude. As seen in Fig. 4a, the beam position 4 is south of the darkest area in the dark spot, where the maximum depletion of electron density occurs and thus, the more intense downward FAC are expected.

\section{Discussion}

\subsection{Stable narrow arc}

In cases (b) and (c), the stable electric field associated with the narrow arc is confined to a spatially restricted region, within $\sim 100 \mathrm{~km}$ from the arc. Based on radar measurements, a number of studies have reported features of arc-associated electric fields. Aikio et al. (1993) showed that the width of an arc-associated electric field was estimated to be less than, or equal to, $45 \mathrm{~km}$. Opgenoorth et al. (1990) suggested that the width of very high perpendicular electric fields, associated with a reduced electron density, was estimated to be of the order of the arc width (about $20 \mathrm{~km}$ ). Lewis et al. (1994) estimated the horizontal extent of the electric field to be about $40 \mathrm{~km}$. It is also noted that the arc-associated electric field shown by Opgenoorth et al. (1990) or Lewis et al. (1994) accompanies a reduction of the electron density, while our observed arc-associated electric field in cases (b) and (c) is not accompanied with such a strong reduction. Past studies showed that the arc-associated electric field was observed only on one side of the narrow arc. Baumjohann (1983) has explained that the ambient electric field is northward (southward) directed in the evening (morning) sector and thus, the arc-associated meridional electric field must be anti-symmetric with respect to the southern (northern) border of the arc, being northward (southward) directed in a region a few kilometers south (north) of the arc and southward (northward) inside the arc. However, our observation in cases (b) and (c) exhibits the arc-associated electric field on both the northward and southward sides outside the narrow arc. On both sides, the arc-associated electric field directs towards the narrow arc in a spatially restricted region. This feature is rather similar to that of the event shown by Burch et al. (1976), who determined the characteristics of pairs of oppositely directed spikes in ionospheric convection velocities using data from AE-C spacecraft. Their convection velocities are associated with the inverted- $\mathrm{V}$ type auroral electron precipitation. They suggested that the relationship between the electron precipitation and the electric field spikes was consistent with an upward-flowing FAC that was fed by Pedersen currents from the adjacent regions of strong convection.

In Fig. 7, for cases (b) and (c), $\Sigma_{P} \operatorname{div} \boldsymbol{E}$ produces an upward FAC, while $\boldsymbol{E} \cdot \operatorname{grad} \Sigma_{P}$ produces an opposed downward FAC. Sato et al. (1995) have reported a similar situation at the equatorward edge of the large-scale downward region 1 current. A small rot $\boldsymbol{E}$ in Fig. 7 for cases (b) and (c) shows that the temporal variation is small. However, it is suspected that the region observed with the EISCAT radar includes both upward and downward FAC regions in these cases. As described by Marklund (1984), if a transverse height-integrated current has a difference between two observed positions, there is a FAC between the two. For example, in Fig. 6c, the Pedersen current in the west or east position is larger $(\sim 150 \mathrm{~mA} / \mathrm{m})$ than that in the south or north position $(\sim 50 \mathrm{~mA} / \mathrm{m})$. This implies that a northwestward Pedersen current $(\sim 150 \mathrm{~mA} / \mathrm{m})$ flows in the center of the four observed positions. Thus, it is expected that FACs flow upward in the northern half of the four positions, and downward in the southern half. Similarly in Fig. 6b, a southeastward Pedersen current is expected to flow in the center of the four positions, with an upward FAC in the northern half and a downward FAC in the southern half. Thus, we conclude that the spatial scale of the region observed with the EISCAT radar was too large in comparison to the spatial current structure, in order to apply formula (2) reliably for cases (b) and (c). In these cases, we cannot confirm whether $\Sigma_{P}$ $\operatorname{div} \boldsymbol{E}$ and $\boldsymbol{E} \cdot \operatorname{grad} \Sigma_{P}$ really produce FACs with opposing directions, as reported by Sato et al. (1995).

\subsection{Stable broad arc}

As shown in Fig. 7, for cases (d) and (e), a downward FAC is inferred to exist north of the arc, and an upward FAC inside the broad arc. In cases (d) and (e), both $\Sigma_{P}$ $\operatorname{div} \boldsymbol{E}$ and $\boldsymbol{E} \cdot \operatorname{grad} \Sigma_{P}$ produce the same direction FAC. Only in the north-south direction, in case (e), $\Sigma_{P} \operatorname{div} \boldsymbol{E}$ and $\boldsymbol{E} \cdot \operatorname{grad} \Sigma_{P}$ produce FACs with opposing directions, in agreement with the result by Sato et al. (1995). On the other hand, $(\boldsymbol{b} \times \boldsymbol{E}) \cdot \operatorname{grad} \Sigma_{H}$ is small and not effective in closing the FAC.

In Fig. 4e, the aurora moves slightly westward with the passage of time, keeping its form, and is favorable for our radar mode, taking into account the Earth's rotational effect. Also in case (e), the area scanned by the beam positions 58 is small (see Fig. 2) and the calculated rot $\boldsymbol{E}$ is extremely small (see Fig. 7). Therefore, the situation in case (e) is the most appropriate one out of all our cases, in order to calculate a FAC using formula (2). The calculated FAC is also the most reliable in case (e) rather than that in any of the other cases. The upward FAC density by $\Sigma_{P} \operatorname{div} \boldsymbol{E}$ and $\boldsymbol{E} \cdot \operatorname{grad} \Sigma_{P}$ are $\sim 1.3$ and $\sim 0.7 \mu \mathrm{A} / \mathrm{m}^{2}$, respectively, and thus the contribution of $\Sigma_{P} \operatorname{div} \boldsymbol{E}$ is nearly twice as large as that of $\boldsymbol{E} \cdot \operatorname{grad} \Sigma_{P}$ in case (e).

These results are consistent with the results by Kosch et al. (2000) and Kosch and Nielsen (2001). However, they discussed a plasma vortex associated with a black aurora, which seems similar to the dark spot shown in case (a). Our results are based on observations of the stable arc around 02:00 UT $(\sim 05: 00$ MLT) in cases (d) and (e). We have also checked several auroral images and could find auroral forms with the same features in the early morning on other days (not shown here). Thus, the arc in cases (d) and (e) is considered to be a common feature seen in the early morning. Unfortunately, the electric fields in these cases are not very large, and thus, it is difficult for STARE to measure reliable electric fields 

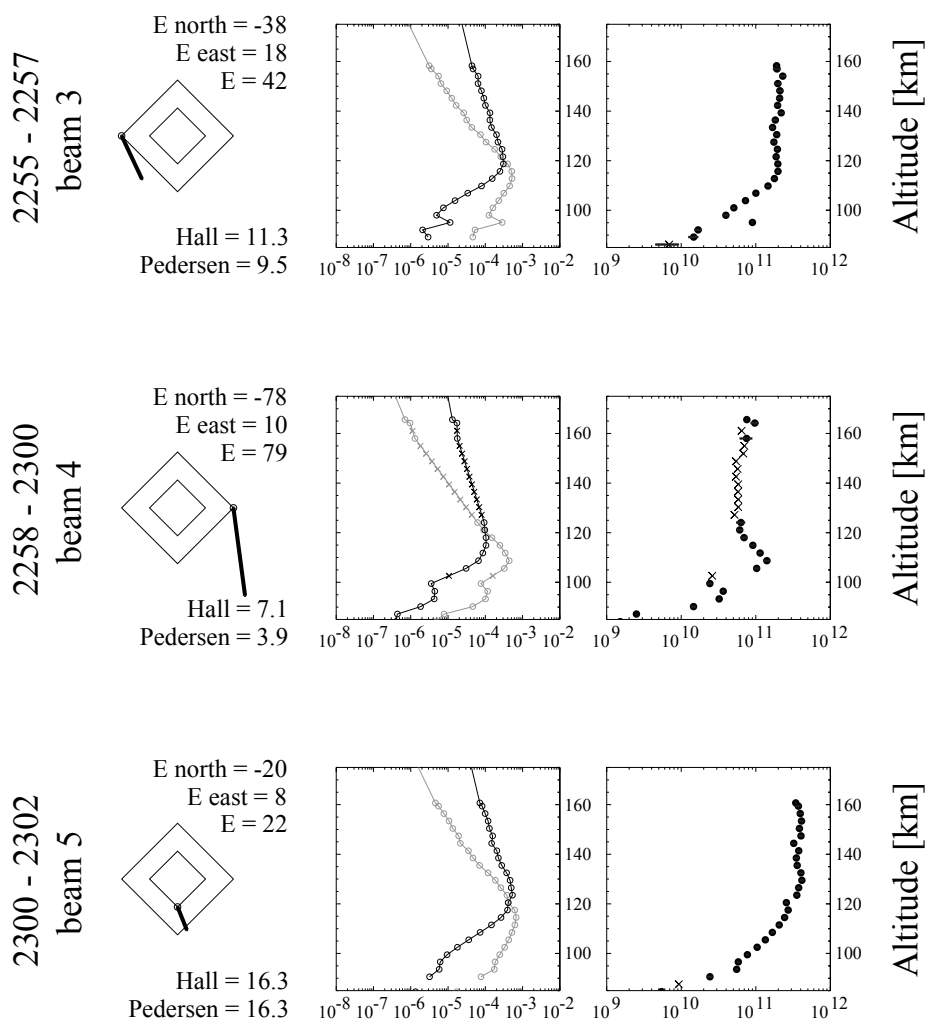

Electric Field $[\mathrm{mV} / \mathrm{m}]$

Conductance $[\mathrm{S}]$
$[\mathrm{S} / \mathrm{m}]$

Conductivity $\left[/ \mathrm{m}^{3}\right]$

Electron Density
Fig. 10. EISCAT radar measurements of the altitude profile of the Pedersen (thick line) and Hall (thin line) conductivities and the electron density, around the time when a dark spot was observed. and to deduce the FAC density, as demonstrated by Kosch et al. (2000).

Two major high-latitude FAC systems have been statistically shown to exist (Iijima and Potemra, 1976). In the dawn sector, the poleward currents, denoted as region 1 currents, flow into the ionosphere, and the equatorward currents, denoted as region 2 currents, flow away from the ionosphere. This distribution is consistent with our calculated FAC in cases (d) and (e), which is downward north of the stable arc, and upward to the south. In Fig. 3, the ground magnetometer observation around 02:00 UT indicates $\Delta Z \sim 0$, meaning that the electrojet current center may be located near Troms $\varnothing$. In the morning sector, Senior et al. (1982) and Sato et al. (1995) have inferred that the center of the westward electrojet current is located near the region $1 /$ region 2 FAC reversal. Thus, the ground magnetometer data showing $\Delta Z \sim 0$ are consistent with our calculated FAC distribution. The estimated FAC densities $\left(\sim 2 \mu \mathrm{A} / \mathrm{m}^{2}\right)$ have the same order as the reported FAC densities by satellite observations (e.g. Iijima and Potemra, 1978; Fukunishi et al., 1993).

In Fig. 5, except in case (a), all of the stable arcs have a conductivity gradient in the east-west direction, and the conductivity in the east position is higher than that in the west position in each case. Also in Fig. 4, the stable arcs are brighter towards the east. This indicates that particle precip- itation, which is effective in enhancing ionospheric conductivities, should be greater going from midnight to the dawn side. As mentioned before, we see similar stable arcs in aurora images on other days, and the stable arcs in Fig. 4 are thus considered to be a common feature around these MLTs.

In the ionosphere, the conductivity is well determined by the precipitating electron energy and electron energy flux (e.g. Spiro et al., 1982; Robinson et al., 1987; Hardy et al., 1987). The conductivity gradient in the north-south direction plays a crucial role in producing the FAC rather than that in east-west direction, as mentioned before. On the other hand, in the magnetosphere, the plasma pressure is contributed mainly by hot ions (e.g. Spence et al., 1989). In the magnetosphere, a pressure-gradient force on the plasma in the equatorial plane plays a crucial role on FAC generation (e.g. Iijima, 2000).

\subsection{Narrow bright arc and dark spot}

In case (a), we see a region with a very high electric field adjacent to a narrow bright arc. In the auroral images, this region is identified as a dark spot. The region is estimated to have a spatial dimension, over $150 \times 150 \mathrm{~km}$, that is much larger than that reported for "black auroras" (see a brief review by Kosch et al., 1998b). This implies that our observed 
dark spot may be different from phenomena of black auroras. According to the definition, the black aurora is a lack of emission in a small, well-defined region within an otherwise uniform, diffuse background or within auroras exhibiting a degree of shear behavior intermediate between that of diffuse and discrete aurora (see Trondsen and Cogger, 1997). Black auroras appear to be a common feature of the late recovery phase of an auroral substorm. Several high spatial and temporal resolution optical observations have identified black auroras. Schoute-Vanneck et al. (1990) measured 40 black filaments to find a typical width of $1 \mathrm{~km}$, and length of 5-20 km. Trondsen and Cogger (1997) measured 31 black auroral patches and arc segments and found a typical width of $0.5-1.5 \mathrm{~km}$.

On the other hand, the plasma vortex analyzed by Kosch et al. (2000) has a spatial dimension, $200 \times 200 \mathrm{~km}$, which is of the same order as our observed dark spot. If the dark spot is a similar feature of the plasma vortex shown by Kosch et al. (2000), a downward FAC is expected in the dark spot. Indeed, our observed depletion of electron density over $110 \mathrm{~km}$ altitude implies that the dark spot occurs in the downward FAC, which carries ionospheric electrons into the magnetosphere as a current carrier. As mentioned before, the position observed by the beam 4 is on the south side of the darkest point in the dark spot, where the greatest depletion of electron density occurs and thus, the more intense downward FAC is expected. In the case analyzed by Kosch et al. (2000), the strongest downward FAC in the center of the plasma vortex is also far from the bright arc $(\sim 100 \mathrm{~km})$.

There may be an alternative explanation for the electron density depletion within the dark spot. High electric fields can alter the ion composition and also lead to an electron density decrease due to enhanced recombination. According to the result by Schunk et al. (1975), this decrease is seen mainly in the F-region, however, it is noted that the density depletion we detected within the dark spot is seen also in the E-region (above $110 \mathrm{~km}$ ).

When the dark spot is identified, a narrow bright arc appears simultaneously south of the dark spot. Aikio et al. (1993) have reported the simultaneous intensification of the arc-associated electric field and the optical brightening of the auroral arc. Due to the beam swing, the EISCAT radar could observe both inside the dark spot and the narrow arc. During 23:00-23:02 UT, the EISCAT radar sounded inside the narrow arc with the beam position 5. Both the Pedersen and Hall conductances increase with a smaller electric field. Moving from inside the dark spot to inside the narrow arc, the height-integrated Pedersen current does not change much. In our case (a), the electric field points from the dark spot into the narrow arc; thus, one can conclude that the Pedersen current is the closure current between the dark spot and the narrow arc. Using the observed conductance and the electric field, we can quantitatively estimate the height-integrated Pedersen and Hall currents; 310 and $560 \mathrm{~mA} / \mathrm{m}$ inside the dark spot during 22:58-23:00 UT, and 360 and $360 \mathrm{~mA} / \mathrm{m}$ inside the narrow arc during 23:00-23:02 UT, respectively. The difference in the Pedersen and Hall currents between the two regions is 50 and $200 \mathrm{~mA} / \mathrm{m}$, respectively. It should be noted, however, that these values have some ambiguity, because there is a time lag between observations of the two regions. The dark spot became fainter when the EISCAT radar measured inside the narrow arc during 23:00-23:02 UT. This may imply that the current morphology changes. Also, inside the narrow arc, the conductivities and the electric field are not measured on the same magnetic field line by the beam position 5. This may add an error to the calculated current inside the arc. However, this difference in the Pedersen current is smaller than that of about $100 \mathrm{~mA} / \mathrm{m}$ in case (b).

\subsection{EISCAT radar mode employed and future}

The neutral wind cannot be measured in our observations, and is neglected in the present analysis. In the ionosphere, the electric field is the sum of the potential electric field (derived from the plasma motion in the F-region) and the neutral dynamo electric field $\boldsymbol{U} \times \boldsymbol{B}$, where $\boldsymbol{U}$ is the neutral wind velocity. The neutral wind velocity varies greatly with altitudes in the E-region (e.g. Nozawa and Brekke, 1995); thus, the dynamo electric field $\boldsymbol{U} \times \boldsymbol{B}$ is also highly altitude dependent. The amplitude of the dynamo electric field occasionally becomes comparable to that of the potential electric field (Fujii et al., 1998, 1999). Thus, especially in the case with temporal variations, we may not ignore the contribution of the neutral wind and the dynamo electric field. A more detailed analysis considering the distribution of the neutral wind and $\operatorname{div}(\boldsymbol{E}+\boldsymbol{U} \times \boldsymbol{B})$ will be needed for a better understanding.

It should be noted that the EISCAT radar data and the analysis inevitably include errors (see Fujii et al., 1998). Each measured parameter has an error at the time of the measurement and the data processing. The errors of the ion velocity can provide uncertainties for the electric field, less than $\pm 3 \mathrm{mV} / \mathrm{m}$ for quiet periods and less than $\pm 6 \mathrm{mV} / \mathrm{m}$ during high electric field conditions in our experiment. The average error of the electron density is about $\pm 6 \%$ and can provide uncertainties for the conductivity. However, it is not possible to evaluate errors of the conductivity quantitatively. The difference between the real neutral atmosphere and the model atmosphere used (MSISE-90) may also give significant errors to the ion-neutral or electron-neutral collision frequencies and thereby to the conductivities, but we do not have the means to estimate these uncertainties.

In the mode employed, the EISCAT radar alternately observed at two different scales; 100 or $50 \mathrm{~km}$ at $240 \mathrm{~km}$ altitude. The smaller one is sufficient to detect differences in ionospheric conductivities and electric fields, while the larger one seems too large for several auroral features. Also, the conductance is determined across magnetic field lines when the transmitting antenna is not directed along the magnetic field line. For example, with the lowest elevation beam 1, the horizontal scale is $\sim 2 \mathrm{~km}$ for the height range of $10 \mathrm{~km}$. This may also add an error to the calculated conductance, especially for the narrow arc. The smaller the spatial scale for measurements is, the smaller this error becomes. 
In the future, using a mode in which the EISCAT radar sounds on a smaller spatial scale as quickly as possible, we will investigate the closure of FACs also in the evening sector. As illustrated by Senior et al. (1982), the distribution of auroral precipitation and electric fields in the evening sector is different from that in the morning sector investigated in present study. Fujii et al. (1990) suggested that the conductivity gradient controlled significantly the amplitude of the FAC density and that $\boldsymbol{E} \cdot \operatorname{grad} \Sigma_{P}$ worked more effectively in comparison with $\Sigma_{P} \operatorname{div} \boldsymbol{E}$ in the evening sector. The ionospheric current intensity in the evening sector is characterized by a relatively strong electric field (e.g. Davies and Lester, 1999), and this situation is favorable for observations with STARE, which can obtain the distribution of electric fields in a wider area. The EISCAT radar is capable of providing the conductivity information; thus, a simultaneous study with STARE and satellites will allow us not only to examine the validity of our FAC derivation, but also to better understand the ionospheric roles on the magnetosphereionosphere coupling.

\section{Summary}

We have conducted special experiments with the EISCAT radar on 9 October 1999, in an attempt to derive FACs from the spatial distributions of ionospheric currents, in order to understand the ionospheric current closure of FACs. The results are as follows:

1. If the ionosphere is stable during the time periods concerned, rot $\boldsymbol{E}$ becomes relatively small and justifies our approximation.

2. The electric field associated with a narrow arc was confined in a spatially restricted region, within $\sim 100 \mathrm{~km}$ from the arc, with no distinct depletion of electron density. This electric field was directed towards the arc on both the northward and southward sides.

3. Inside a stable broad $\operatorname{arc}, \Sigma_{P} \operatorname{div} \boldsymbol{E}$ and $\boldsymbol{E} \cdot \operatorname{grad} \Sigma_{P}$ produced FACs with the same direction around 05:00 MLT, when the EISCAT radar presumably crossed the boundary between the large-scale upward and downward current regions. In the most successfully observed case, the contribution of $\Sigma_{P}$ div $\boldsymbol{E}$ is nearly twice as large as that of $\boldsymbol{E} \cdot \operatorname{grad} \Sigma_{P}$. On the other hand, the contribution of $(\boldsymbol{b} \times \boldsymbol{E}) \cdot \operatorname{grad} \Sigma_{H}$ was small and not effective in closing FACs.

4. During the recovery phase of a substorm, a region of very high electric field was observed adjacent to a narrow bright arc within the large-scale FACs. The Pedersen current is the closure current across the narrow arc. In the region of the very high electric field, the electron density is depleted above $110 \mathrm{~km}$ altitude. In the auroral images, this region is identified as a dark spot with a spatial scale of over $150 \times 150 \mathrm{~km}$. The dark spot and the electron depletion were likely in existence for a limited time of a few minutes.

Acknowledgements. We are indebted to the director and staff of EISCAT for operating the facility and supplying the data. EISCAT is an international association supported by Finland (SA), France (CNRS), the Federal Republic of Germany (MPG), Japan (NIPR), Norway (NFR), Sweden (NFR) and the United Kingdom (PPARC). Thanks also to the staff of WDC-C2, Kyoto University for providing geomagnetic indices. The IMAGE data was kindly supplied by the Auroral Observatory, University of Troms $\emptyset$.

Topical Editor M. Lester thanks U. P. Lovhaug and K. Schlegel for their help in evaluating this paper.

\section{References}

Aikio, A. T., Opgenoorth, H. J., Persson, M. A. L., and Kaila, K. U.: Ground-based measurements of an arc-associated electric field, J. Atmos. Terr. Phys., 55, 797-803, 1993.

Amm, O.: Method of characteristics in spherical geometry applied to a Harang discontinuity situation, Ann. Geophysicae, 16, 413424, 1998.

Barton, C. E.: International geomagnetic reference field: The seventh generation, J. Geomag. Geoelectr., 49, 123-148, 1997.

Baumjohann, W.: Ionospheric and field-aligned current systems in the auroral zone: A concise review, Adv. Space. Res., 2, 55-62, 1983.

Brekke. A. and Hall, C.: Auroral ionospheric quiet summer time conductances, Ann. Geophysicae, 6, 361-376, 1988.

Burch, J. L., Lennartsson, W., Hanson, W. B., Heelis, R. A., Hoffman, J. H., and Hoffman, R. A.: Properties of spikelike shear flow reversals observed in the auroral plasma by Atmosphere Explorer C, J. Geophys. Res., 81, 3886-3896, 1976.

Cahill, L. J., Greenwald, R. A., and Nielsen, E.: Auroral radar and rocket double-probe observations of the electric field across the Harang discontinuity, Geophys. Res. Lett., 5, 687-690, 1978.

Caudal, G.: Field-aligned currents deduced from EISCAT radar observations and implications concerning the mechanism that produces region 2 currents, J. Geophys. Res., 92, 6000-6012, 1987.

Collis, P. N.: EISCAT data base for ionospheric modelling: Fregion and topside ionosphere, Adv. Space. Res., 16, 37-46, 1995.

Davies. J. A. and Lester, M.: The relationship between electric fields, conductances and currents in the high-latitude ionosphere: a statistical study using EISCAT data, Ann. Geophysicae, 17, 43$52,1999$.

de la Beaujardière, O., Vondrak, R., Heelis, R., Hanson, W., and Hoffman, R.: Auroral arc electrodynamic parameters measured AE-C and the Chatanika radar, J. Geophys. Res., 86, 4671-4685, 1981.

Folkestad, K., Hagfors, T.. and Westerlund, S.: EISCAT: An updated description of technical characteristics and operational capabilities, Radio Sci., 18, 867-879, 1983.

Fontaine, D. and Peymirat, C.: Large-scale distributions of ionospheric horizontal and field-aligned currents inferred from EISCAT, Ann. Geophysicae, 14, 1284-1296, 1996.

Fujii, R., Hoffman, R. A., and Sugiura, M.: Spatial relationships between region 2 field-aligned currents and electron and ion precipitation in the evening sector, J. Geophys. Res., 95, 18939 $18947,1990$. 
Fujii, R., Nozawa, S., Matuura, N., and Brekke, A.: Study on neutral wind contribution to the electrodynamics in the polar ionosphere using EISCAT CP-1 data, J. Geophys. Res., 103, 14 73114 739, 1998.

Fujii, R., Hoffman, R. A., Anderson, P. C., Craven, J. D., Sugiura, M., Frank, L. A., and Maynard, N. C.: Electrodynamic parameters in the nighttime sector during auroral substorms, J. Geophys. Res., 99, 6093-6112, 1994.

Fujii, R., Nozawa, S., Buchert, S. C., and Brekke, A.: Statistical characteristics of electromagnetic energy transfer between the magnetosphere, the ionosphere, and the thermosphere, J. Geophys. Res., 104, 2357-2365, 1999.

Fukunishi, H., Takahashi, Y., Nagatsuma, T., Mukai, T., and Machida, S.: Latitudinal structures of nightside field-aligned currents and their relationships to the plasma sheet regions, J. Geophys. Res., 98, $11235-11255,1993$.

Greenwald, R. A., Weiss, W., Nielsen, E., and Thomson, N. R.: STARE: A new radar auroral backscatter experiment in northern Scandinavia, Radio Sci., 13, 1021-1039, 1978.

Hardy, D. A., Gussenhoven, M. S., Raistrick, R., and McNeil, W. J.: Statistical and functional representations of the pattern of auroral energy flux, number flux, and conductivity, J. Geophys. Res., 92, 12 275-12 294, 1987.

Hedin, A. E.: Extension of the MSIS thermosphere model into the middle and lower atmosphere, J. Geophys. Res., 96, 1159-1172, 1991.

Hoffman, R. A.: Observations of ionosphere/magnetosphere interactions from the Dynamics Explorer satellites, AGARD, Ionospheric Structure and Variability on a Global Scale and Interactions with Atmosphere and Magnetosphere p. 17 (SEE N9011361 02-46), April, 1989.

Iijima, T. and Potemra, T. A.: Field-aligned currents in the dayside cusp observed by Triad, J. Geophys. Res., 81, 5971-5979, 1976.

Iijima, T. and Potemra, T. A.: Large-scale characteristics of fieldaligned currents associated with substorms, J. Geophys. Res., 83, 599-615, 1978.

Iijima, T.: Field-aligned currents in geospace: substance and significance, in: Magnetospheric Current Systems, (Eds) Ohtani, S., Fujii, R., Hesse, M., and Lysak, R. L., AGU Geophysical Monograph 118, pp. 107-129, 2000.

Kosch, M. J., Nielsen, E., and Hagfors, T.: A new Digital All-Sky Imager experiment for optical auroral studies in conjunction with the STARE coherent radar system, Rev. Sci. Instrum., 69, 578584, 1998a.

Kosch, M. J., Scourfield, N. W. J., and Nielsen, E.: A self-consistent explanation for a plasma vortex associated with the brightening of an auroral arc, J. Geophys. Res., 103, 29 383-29391, 1998 b.

Kosch, M. J., Amm, O., and Scourfield, M. W. J.: A plasma vortex revisited: The importance of including ionospheric conductivity measurements, J. Geophys. Res., 105, 24 889-24 898, 2000.

Kosch, M. J. and Nielsen, E.: Statistical average estimates of high latitude field-aligned currents from the STARE and SABRE coherent VHF radar systems, Adv. Space Res., 27, 1239-1244, 2001.

Kosch, M. J., Scourfield, M. W. J., and Amm, O.: The importance of conductivity gradients in ground-based field-aligned current studies, Adv. Space Res., 27, 1277-1282, 2001.

Küppers, F., Untiedt, J., Baumjohann, W., Lange, K., and Jones, A. G.: A two-dimensional magnetometer array for ground-based observations of auroral zone electric currents during the International Magnetospheric Study (IMS), J. Geophys., 46, 429, 1979.
Lewis, R. V., Williams, P. J. S., Jones, G. O. L., Opgenoorth, H. J., and Persson, M. A. L.: The electrodynamics of a drifting auroral arc, Ann. Geophysicae, 12, 478-480, 1994.

Lysak, R. L.: Electrodynamic coupling of the magnetosphere and ionosphere, Space Sci. Rev., 52, 33-87, 1990.

Marklund, G.: Auroral arc classification scheme based on the observed arc-associated electric field pattern, Planet. Space Sci., 32, 193-211, 1984.

Nozawa, S. and Brekke, A.: Studies of the E-region neutral wind in the disturbed auroral ionosphere, J. Geophys. Res., 100, $14717-$ $14734,1995$.

Opgenoorth, H. J., Häggström, I., Williams, P. J. S., and Jones, G. O. L.: Regions of strongly enhanced perpendicular electric fields adjacent to auroral arcs, J. Atmos. Terr. Phys., 52, 449458, 1990.

Rishbeth, H. and Williams, P. J. S.: The EISCAT ionospheric radar: the system and its early results, Q. J. R. Astron. Soc., 26, 478512, 1985.

Robinson, R. M., Vondrak, R. R., Miller, K., Dabbs, T., and Hardy, D. A.: On calculating ionospheric conductivities from the flux and energy of precipitating electrons, J. Geophys. Res., 92, 2565-2569, 1987.

Sato, M., Kamide, Y., Richmond, A. D., Brekke, A., and Nozawa, S.: Regional estimation of electric fields and currents in the polar ionosphere, Geophys. Res. Lett., 22, 283-286, 1995.

Schlegel, K.: Auroral zone E-region conductivities during solar minimum derived from EISCAT data, Ann. Geophysicae, 6, 129-138, 1988.

Schoute-Vanneck, H., Scourfield, M. W. J., and Nielsen, E.: Drifting black aurorae?, J. Geophys. Res., 95, 241-246, 1990.

Schunk, R. W., Raitt, W. J., and Banks, P. M.: Effect of electric fields on the daytime high-latitude E- and F-regions, J. Geophys. Res., 80, 3121-3130, 1975.

Senior, C., Robinson, R. M., and Potemra, T. A.: Relationship between field-aligned currents, diffuse auroral precipitation and the westward electrojet in the early morning sector, J. Geophys. Res., 87, 10 469-10477, 1982.

Senior, C., Fontaine, D., Caudal, G., Alcaydé, D., and Fontanari, J.: Convection electric fields and electrostatic potential over $61^{\circ}<\Lambda<72^{\circ}$ invariant latitude observed with the European incoherent scatter facility. 2. Statistical results, Ann. Geophysicae, 8, 257-272, 1990.

Senior, C.: Solar and particle contributions to auroral heightintegrated conductivities from EISCAT data: a statistical study, Ann. Geophysicae, 9, 449-460, 1991.

Sofko, G. J., Greenwald, R., and Bristow, W.: Direct determination of large-scale magnetospheric field-aligned currents with SuperDARN, Geophys. Res. Lett., 22, 2041-2044, 1995.

Spence, H. E., Kivelson, M. G., Walker, R. J., and McComas, D. J.: Magnetospheric plasma pressures in the midnight meridian: Observations from 2.5 to $35 R_{E}$, J. Geophys. Res., 94, 52645272, 1989.

Spiro, R. W., Reiff, P. H., and Maher, Jr., L. J.: Precipitating electron energy flux and auroral zone conductance - An empirical model, J. Geophys. Res., 87, 8215-8227, 1982.

Trondsen, T. S. and Cogger, L. L.: High-resolution television observations of black aurora, J. Geophys. Res., 102, 363-378, 1997.

Yasuhara, F., Kamide, Y., and Holt, J. M.: Field-aligned currents in high latitudes estimated from Millstone Hill radar observations of ion drifts, J. Geophys. Res., 87, 2553-2557, 1982. 